\title{
Multiple Wearable Sensors in Parkinson and Huntington Disease Individuals: A Pilot Study in Clinic and at Home
}

\author{
Jamie L. Adams ${ }^{a}$ b Karthik Dinesh ${ }^{c} \quad$ Mulin Xiong $^{b}$ \\ Christopher G. Tarollia, ${ }^{a}$ Saloni Sharma ${ }^{b}$ Nirav Sheth $^{d} \quad$ A.J. Aranyosid ${ }^{d}$ \\ William Zhu ${ }^{b}$ Steven Goldenthal ${ }^{b}$ Kevin M. Biglan ${ }^{a, b} \quad$ E. Ray Dorsey ${ }^{a}$ b \\ Gaurav Sharmac, e \\ a Department of Neurology, University of Rochester Medical Center, Rochester, NY, USA; \\ ${ }^{b}$ Center for Health and Technology, University of Rochester Medical Center, Rochester, NY, \\ USA; ' ${ }^{c}$ Department of Electrical and Computer Engineering, University of Rochester Medical

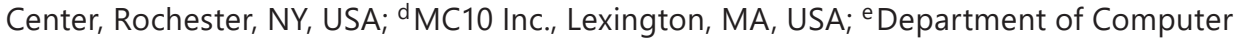 \\ Science, University of Rochester, Rochester, NY, USA
}

\section{Keywords}

Parkinson disease $\cdot$ Huntington disease $\cdot$ Technology $\cdot$ Movement disorders $\cdot$ Remote sensing technology · Monitoring · Ambulatory patients · Clinical study · Gait

\section{Abstract}

Background: Clinician rating scales and patient-reported outcomes are the principal means of assessing motor symptoms in Parkinson disease and Huntington disease. However, these assessments are subjective and generally limited to episodic in-person visits. Wearable sensors can objectively and continuously measure motor features and could be valuable in clinical research and care. Methods: We recruited participants with Parkinson disease, Huntington disease, and prodromal Huntington disease (individuals who carry the genetic marker but do not yet exhibit symptoms of the disease), and controls to wear 5 accelerometer-based sensors on their chest and limbs for standardized in-clinic assessments and for 2 days at home. The study's aims were to assess the feasibility of use of wearable sensors, to determine the activity (lying, sitting, standing, walking) of participants, and to survey participants on their experience. Results: Fifty-six individuals (16 with Parkinson disease, 15 with Huntington disease, 5 with prodromal Huntington disease, and 20 controls) were enrolled in the study. Data were successfully obtained from $99.3 \%$ (278/280) of sensors dispatched. On average, individuals with Huntington disease spent over $50 \%$ of the total time lying down, substantially more than individuals with prodromal Huntington disease $(33 \%, p=0.003)$, Parkinson disease $(38 \%, p=$ 
Adams et al.: Multiple Wearable Sensors in Parkinson and Huntington Disease Individuals

0.01 ), and controls (34\%; $p<0.001$ ). Most (86\%) participants were "willing" or "very willing" to wear the sensors again. Conclusions: Among individuals with movement disorders, the use of wearable sensors in clinic and at home was feasible and well-received. These sensors can identify statistically significant differences in activity profiles between individuals with movement disorders and those without. In addition, continuous, objective monitoring can reveal disease characteristics not observed in clinic.

(C) 2017 The Author(s)

Published by S. Karger AG, Basel

\section{Introduction}

In Social Physics, Pentland [1] argues that studies should measure health on a scale of observations per person per minute. However, current metrics measure health on the period of months or even years. These measures are largely dominated by episodic, rater-dependent assessments that are almost exclusively performed in the clinic [2,3]. Sensors, including wearable accelerometers, can continuously capture data that can be used to assess the health of individuals, and are particularly relevant in those affected by movement disorders. Frequent, objective, and sensitive assessments are critical to measuring health trends and determining the efficacy of novel therapeutics.

Parkinson disease is an increasingly common neurological disorder characterized by rest tremor, slowness in movement, rigidity, and postural instability. Because most of these motor and many nonmotor features (e.g., sleep disturbances) manifest externally, wearable sensors have been used to evaluate Parkinson disease for the last decade [4]. These sensors have extensively evaluated gait, slowness in movement, tremor, speech, activity, sleep disturbances, and autonomic dysfunction [5]. However, current limitations in the field include narrow spatial resolution from the sensors, limited analytical methods, and cumbersome devices [5].

Huntington disease is a rare genetic disorder that is characterized by involuntary movements (principally chorea, which are hyperkinetic, unpredictable movements), behavioral changes, and cognitive impairment [6]. The onset of the disease typically occurs in individuals who are in their 30 s and 40 s with a long asymptomatic or minimally symptomatic prodromal period before diagnosis. Unlike Parkinson disease, there have been few studies of wearable sensors for Huntington disease with only two studies recently published $[7,8]$, and only one of which evaluated recordings from home [8]. Moreover, no studies have examined the prodromal period in Huntington disease, which is an area of unmet need [2].

To address these gaps in our knowledge, we conducted a study of individuals with Parkinson disease, Huntington disease, or prodromal Huntington disease, and individuals without a movement disorder. We used 5 wearable and flexible sensors in the clinic and at home to evaluate the feasibility of capturing data from these sensors, characterize participant activities (proportion of day spent lying, sitting, standing, or walking), and survey participants on their experience.

\section{Materials and Methods}

\section{Study Overview and Design}

We conducted a 2-day observational study using accelerometer-based BioStampRC ${ }^{\circledR}$ wearable sensors developed by MC10 Inc. (Lexington, MA, USA) [9] in individuals with Parkinson disease, Huntington disease, or prodromal Huntington disease, and in controls 
Fig. 1. Study participant wearing chest, thigh, and forearm sensors.

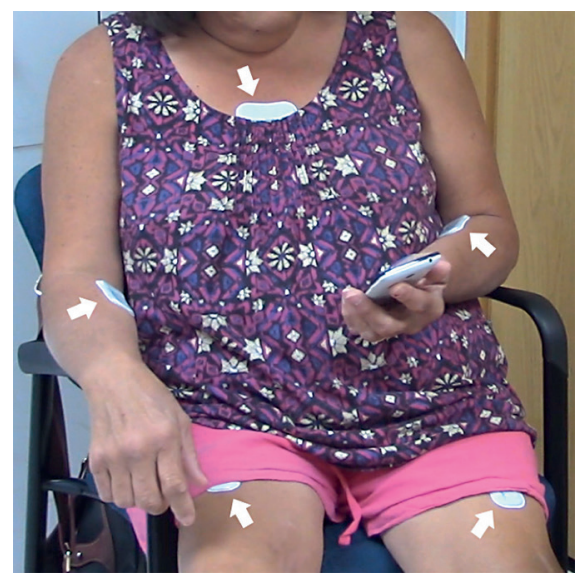

without movement disorders. The goals of this study were to: (1) determine the feasibility of capturing data from structured motor tasks and passive monitoring of individuals with movement disorders using wearable sensors; (2) assess the activity (lying, sitting, standing, walking) of study participants in everyday life; and (3) evaluate participant experience with the sensors via survey. All participants gave informed written consent prior to participation in the study and all procedures were approved by the University of Rochester's institutional review board.

Participants with Parkinson disease and Huntington disease had self-reported clinical diagnoses and participants with prodromal Huntington disease had confirmatory genetic testing (CAG trinucleotide expansion of at least 36 repeats) but did not have sufficient motor signs to warrant a diagnosis of manifest Huntington disease. All participants were recruited from local and regional support groups, clinics, and study interest registries. Unaffected and age-matched family, friends, and community members were recruited as controls. Prior to the clinical visit, the full written consent form was provided to potential participants for review.

In clinic, participants underwent the Montreal Cognitive Assessment [10] and signed the written consent form and video waiver form before providing basic demographic data, medical history, and information on technology use. Five sensors were then adhered to each participant using double-sided adhesives, with one on each anterior thigh, one on each anterior forearm, and one on the chest (Fig. 1). Participants were video-recorded undergoing standard clinical assessments including the Movement Disorder Society - Unified Parkinson Disease Rating Scale [11], the Unified Huntington Disease Rating Scale [12], Timed Up and Go [13], Ten-Meter Walk [14], and smartphone assessments [15]. Following the clinical visit, participants wore the sensors at home for an additional continuous interval totaling approximately 48 hours (sensors could be worn in the shower and during sleep). During this time, participants filled out an activity diary recording their predominant activity for every hour of the day (e.g., walking, sleeping, sitting) and movement disorder medication schedule. Upon completion of the at-home recording period, participants mailed the sensors and diaries to the research team.

Survey

At the study's conclusion, participants completed an electronic survey on their experience with the comfort, security of adhesion, and removal of the sensors (see online suppl. Appendix A; for all online suppl. material, see www.karger.com/doi/10.1159/000479018). 
Adams et al.: Multiple Wearable Sensors in Parkinson and Huntington Disease Individuals

Participants also provided feedback on the perceived value, drawbacks, and potential improvements for the use of wearable sensors. Responses from the survey were analyzed descriptively with response percentages calculated for multiple-choice questions. Open responses to survey questions were reviewed for overall themes.

\section{Analysis}

Sensor Data Collected

Triaxial acceleration data were collected from the sensors at a sampling rate of $31.25 \mathrm{~Hz}$. Activity state information was determined based on sensor data from the chest sensor and one of the two thigh sensors. Analysis of other sensor data including the arm sensors is ongoing and is not reported here.

\section{Activity State Analysis}

Using data from the chest and thigh sensors, intervals were identified when the participant was lying down, sitting, or upright in posture. Further analysis performed on the recorded data in intervals of upright posture was used to differentiate between walking and standing. The chest and thigh sensors were affixed such that, for a participant standing still, the acceleration due to gravity (denoted by $1 \mathrm{~g}$ ) was nominally pointing in the positive $y$ and negative $x$ directions, respectively, given the chosen reference coordinate system. Deviations from these reference orientations, caused by changes in sensor position or associated body part orientation, were corrected by applying a rotation matrix, estimated from the in-clinic "balance test" where the participant stood upright and stationary. Specifically, for the chest sensor, the axes $u$ and angle theta $\theta$ for the rotation that mapped the normalized mean "balance test" acceleration $=\left[x_{c h} y_{c h} z_{c h}\right]$ to the reference value $r_{c}=\left[\begin{array}{lll}0 & 1 & 0\end{array}\right]$ are obtained as follows [16]:

$$
u=\frac{a_{c} \times r_{c}}{\left\|a_{c} \times r_{c}\right\|}, \quad \theta=\cos ^{-1}\left(a_{c} \cdot r_{c}\right) .
$$

From this, the corresponding rotation matrix for remapping the measured data can be computed as follows [16]:

$$
R=\left[\begin{array}{lll}
\cos \theta+u_{x}^{2}(1-\cos \theta) & u_{x} u_{y}(1-\cos \theta)-u_{z} \sin \theta & u_{x} u_{z}(1-\cos \theta)-u_{y} \sin \theta \\
u_{y} u_{x}(1-\cos \theta)+u_{z} \sin \theta & \cos \theta+u_{y}^{2}(1-\cos \theta) & u_{y} u_{z}(1-\cos \theta)-u_{x} \sin \theta \\
u_{z} u_{x}(1-\cos \theta)+u_{y} \sin \theta & u_{z} u_{y}(1-\cos \theta)+u_{x} \sin \theta & \cos \theta+u_{z}^{2}(1-\cos \theta)
\end{array}\right] .
$$

The rotation matrix for the thigh sensor was similarly determined using the corresponding reference orientation and normalized mean "balance test" acceleration.

Recorded data were partitioned into nonoverlapping 5-second intervals and a posture was identified for each interval by determining the dominant axis, i.e., the axis direction along which the mean acceleration was largest. The combination of dominant axes for the chest and thigh sensors was mapped to the posture categories lying down, sitting, and upright according to online supplementary Table $1(<1 \%$ of the data corresponded to the anomalous "upsidedown" posture categories listed in the table; these were omitted from further analysis).

The intervals of upright posture were split into periods corresponding to walking and standing. Walking durations were identified using a normalized autocorrelation-based analysis [17]. The normalized autocorrelation ranges between -1 and 1 and assesses the similarity between the recorded acceleration profile and a temporally delayed version of the same, for a range of temporal delays, or lags. A value of 1 indicates complete similarity, i.e., repetition of the same acceleration profile at the delay defined by the lag, a value of 0 indicates no similarity, and a value of -1 indicates complete similarity of shape with a 180-degree 
Adams et al.: Multiple Wearable Sensors in Parkinson and Huntington Disease Individuals

Table 1. Baseline characteristics of the study population

\begin{tabular}{|c|c|c|c|c|}
\hline Characteristic & $\begin{array}{l}\text { Controls } \\
(n=20)\end{array}$ & $\begin{array}{l}\text { Parkinson } \\
\text { disease } \\
(n=16)\end{array}$ & $\begin{array}{l}\text { Huntington } \\
\text { disease } \\
(n=15)\end{array}$ & $\begin{array}{l}\text { Prodromal } \\
\text { Huntington } \\
\text { disease }(n=5)\end{array}$ \\
\hline \multicolumn{5}{|l|}{ Demographics } \\
\hline Age, years & $58 \pm 16.2$ & $68 \pm 8.7$ & $55 \pm 10.7$ & $38 \pm 8.6$ \\
\hline Sex (women), \% & 70 & 44 & 20 & 40 \\
\hline Ethnicity (white), \% & 90 & 94 & 100 & 100 \\
\hline Hispanic ethnicity, \% & 0 & 0 & 0 & 0 \\
\hline Education (4-year college degree or higher), $\%$ & 50 & 100 & 53 & 40 \\
\hline Currently employed or student, \% & 45 & 19 & 13 & 60 \\
\hline Currently married or in a domestic partnership, \% & 85 & 100 & 53 & 0 \\
\hline \multicolumn{5}{|l|}{ Disease characteristics } \\
\hline Years since diagnosis & NA & $4.9 \pm 4.2$ & $8.2 \pm 4.9$ & NA \\
\hline $\begin{array}{l}\text { Unified Huntington's Disease Rating Scale - } \\
\text { total motor score }(0-124)^{\mathrm{a}}\end{array}$ & $1.7 \pm 2.3$ & NA & $42.7 \pm 10.9$ & $3.4 \pm 4.4$ \\
\hline $\begin{array}{l}\text { Unified Huntington's Disease Rating Scale - } \\
\text { total maximal chorea score }(0-28)^{\mathrm{a}}\end{array}$ & $0.0 \pm 0.0$ & NA & $11.9 \pm 3.0$ & $1.8 \pm 3.5$ \\
\hline Movement Disorder Society - Unified Parkinson's D & & & & \\
\hline Rating Scale - total motor score $(0-132)^{\mathrm{a}}$ & $1.9 \pm 2.1$ & $20.7 \pm 8.3$ & NA & NA \\
\hline Timed Up and Go, s & $7.8 \pm 1.8$ & $10.5 \pm 2.8$ & $11.1 \pm 5.2$ & $7.6 \pm 2.1$ \\
\hline 10-m Walk Test, s & $3.9 \pm 0.7$ & $4.7 \pm 1.1$ & $5.4 \pm 2.0$ & $3.6 \pm 0.6$ \\
\hline Montreal Cognitive Assessment score $(0-30)^{\mathrm{b}}$ & $28.1 \pm 1.4$ & $27.3 \pm 2.2$ & $19.9 \pm 3.8$ & $27.0 \pm 2.5$ \\
\hline \multicolumn{5}{|l|}{ Internet and technology use } \\
\hline Used Internet or email, \% & 100 & 100 & 73 & 100 \\
\hline Used Internet to look up health information, \% & 90 & 100 & 53 & 80 \\
\hline Used smartphone or tablet to access Internet, \% & 95 & 94 & 53 & 100 \\
\hline Used computer or other device to video chat, \% & 100 & 88 & 40 & 60 \\
\hline
\end{tabular}

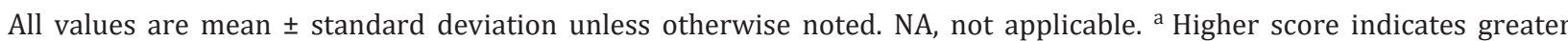
disability. ${ }^{\mathrm{b}}$ Higher score indicates greater cognitive function, score $\geq 26$ is "normal."

change in direction. Periodic steps during normal walking imply a corresponding (quasi) periodicity in the acceleration profile and are therefore manifested in the normalized autocorrelation as lags for which the autocorrelation exhibits a strong peak. Walking durations were identified as epochs within the upright posture intervals for which, over lags between 0.3 and 1.2 seconds that represent the plausible range of step durations, the maximum value of the normalized autocorrelation exceeded 0.1 . The video logs for the in-clinic observations were used to validate this procedure for identifying walking intervals.

\section{Results}

\section{Study Population and Feasibility}

Sixteen participants with Parkinson disease, 15 with Huntington disease, 5 with prodromal Huntington disease, and 20 controls completed the 2-day study. Table 1 provides the baseline characteristics of the study participants. All participants completed in-clinic assessments and at-home monitoring and none withdrew from the study. Participant data were successfully obtained from 278 (99.3\%) of the 280 sensors dispatched. One chest sensor and one leg sensor were lost in transit, both from participants with Huntington disease. 
.... digltal

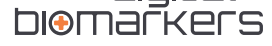

Adams et al.: Multiple Wearable Sensors in Parkinson and Huntington Disease Individuals

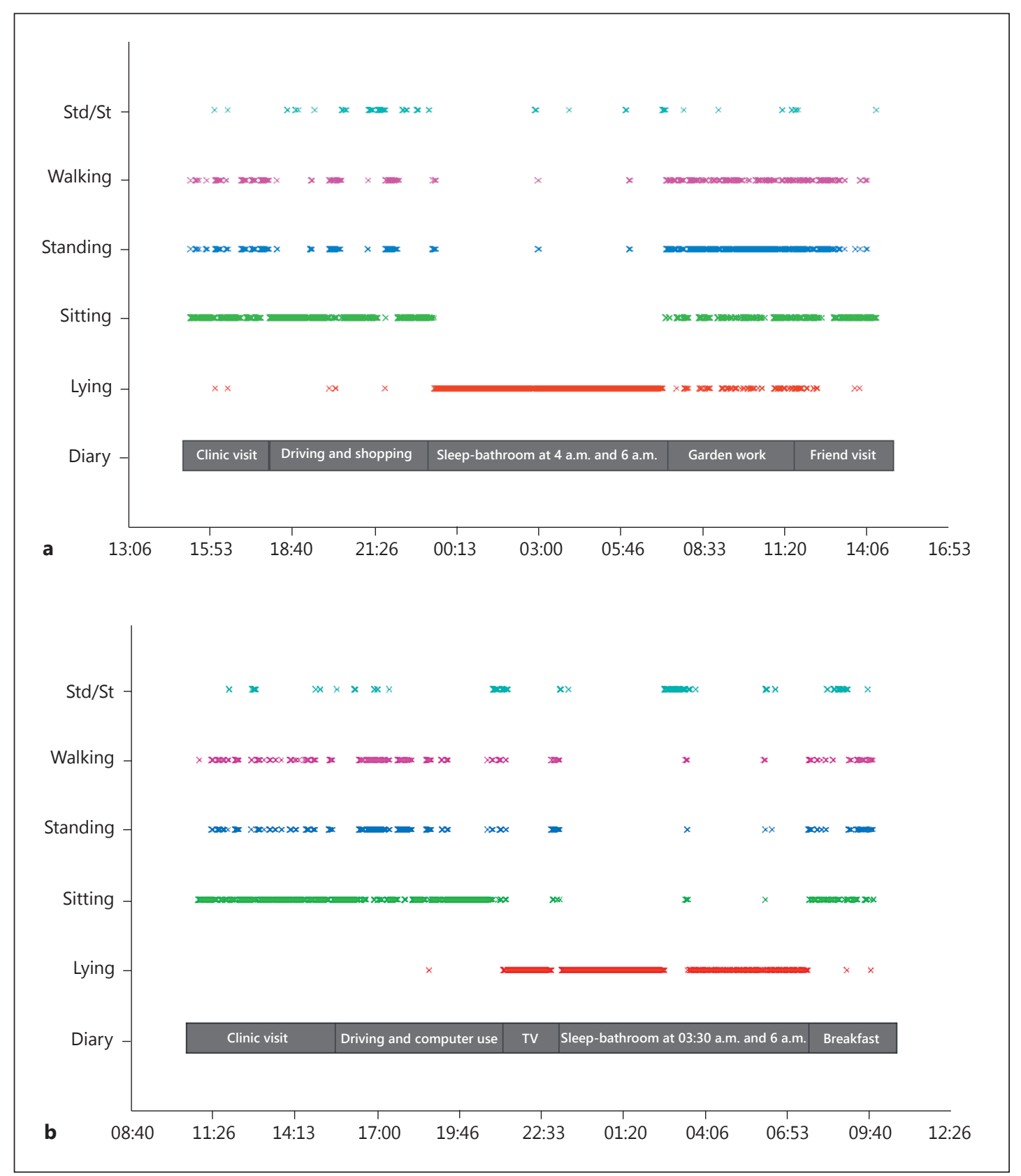

(Figure continued on next page.)

\section{Physical Activity Analysis}

Sensor data collected in clinic and at home were analyzed for activity state and compared to self-reported activity diaries (Fig. 2). Analysis of sensor data collected at home reveals that participants with Huntington disease spend significantly more time lying down compared to control participants $(50.1$ vs. $34.2 \%, p<0.001)$. Figure 3 shows that participants with prodromal Huntington disease and Parkinson disease spend about the same proportion of time lying down as control participants ( $p=0.64, p=0.33$, respectively). Analysis of sleep transitions, which includes changes in lying position (back, side, front) and transitions from lying to sitting or standing, reveal that participants with Parkinson disease $(p=0.011)$, Huntington disease $(\mathrm{p}=0.016)$, and prodromal Huntington disease $(p=0.915)$ all undergo slightly more sleep transitions than control participants (Fig. 4). 


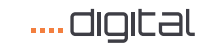

Diømarkers
Digit Biomark 2017;1:52-63

Adams et al.: Multiple Wearable Sensors in Parkinson and Huntington Disease Individuals

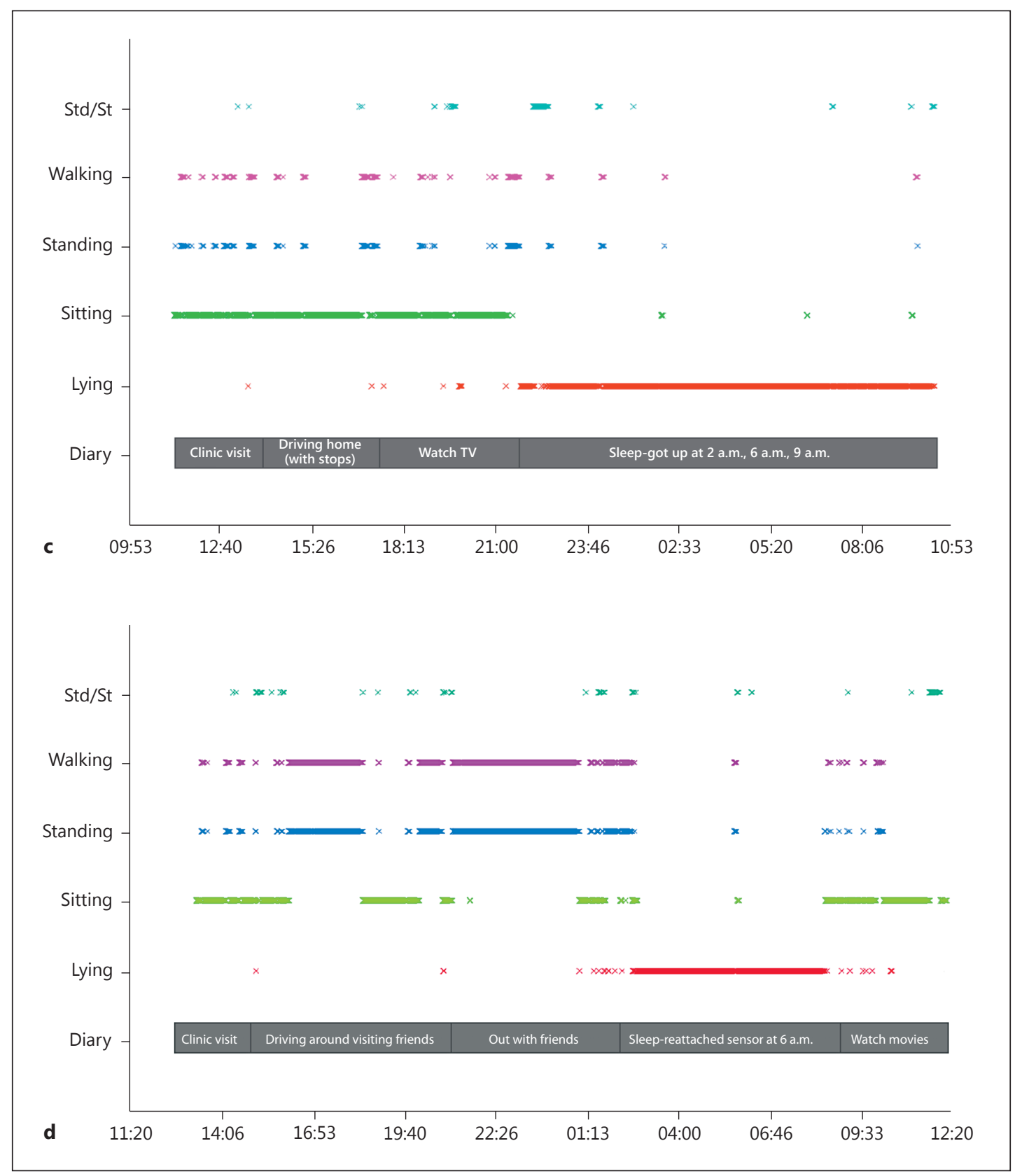

Fig. 2. 24-h sample intervals for representative individuals from each group. a Control participant from day 1, 3 p.m. until day 2, 3 p.m. b Participant with Parkinson disease from day 1, 10 a.m. until day 2, 10 a.m. c Participant with Huntington disease from day 1, 11 a.m. until day 2, 11 a.m. d Participant with prodromal Huntington disease from day 1, 12 p.m. until day 2, 12 p.m. Std, stand; St, sit.

\section{Participant Feedback}

The post-study survey was completed by all 56 participants. As shown in Figure 5, the majority of participants found the sensors to be "comfortable" $(n=42)$ and "easy to remove" $(n=50)$. The most common complaint $(n=31)$ was regarding the adhesion of the sensors and participants reported sensors falling off or coming loose as a major drawback. When asked if they would be willing to use these sensors if asked in the future, $86 \%(n=48)$ of participants said they would be "willing" or "very willing." 
Adams et al.: Multiple Wearable Sensors in Parkinson and Huntington Disease Individuals

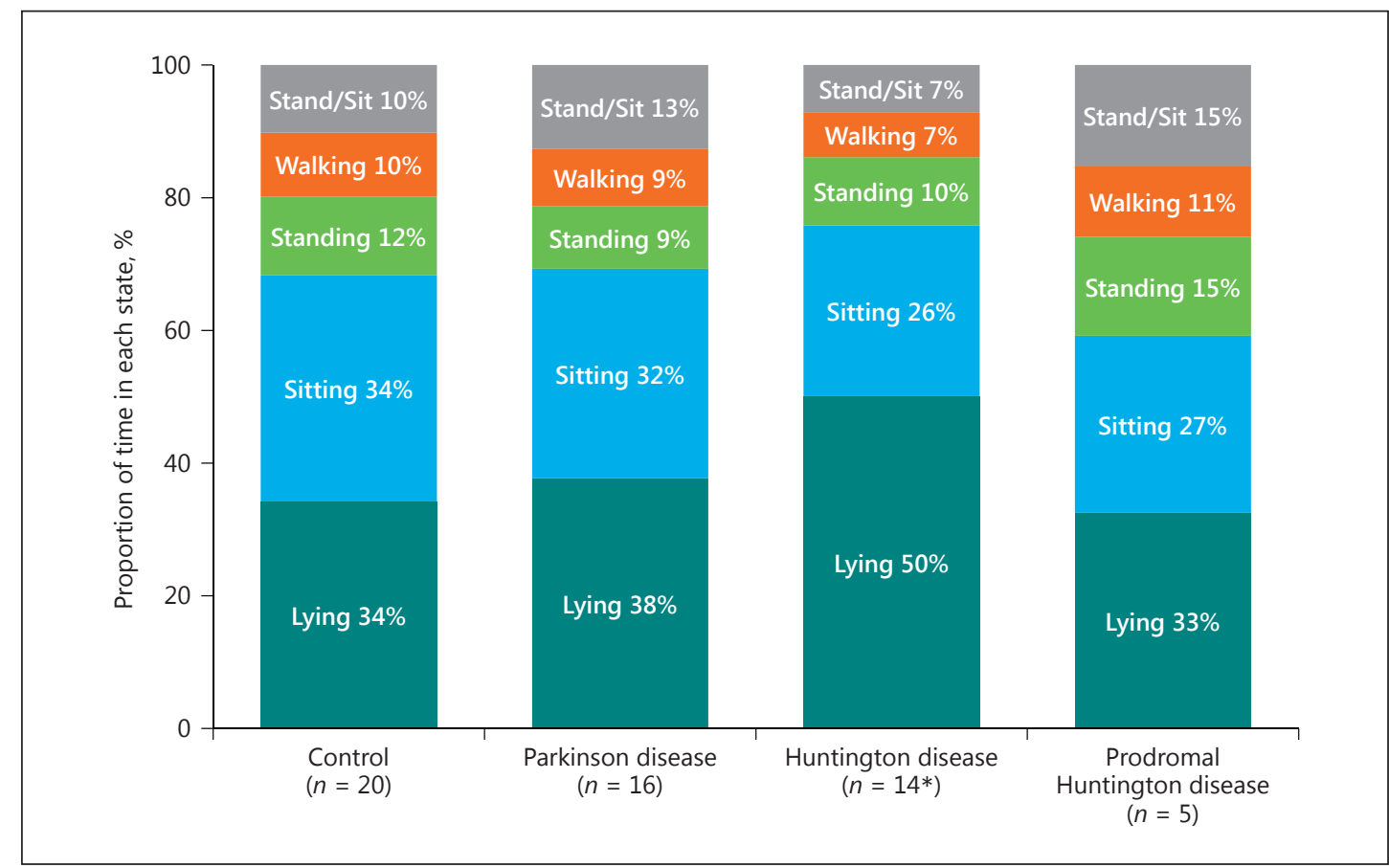

Fig. 3. Proportion of the day participants spent lying down, sitting, standing, and walking. * One participant with Huntington disease lost the chest sensor and could not be included.

Fig. 4. Number of sleep transitions per hour, including changes in lying position and transitions from lying to sitting or standing. * One participant with Huntington disease lost the chest sensor and could not be included. Red lines denote medians.

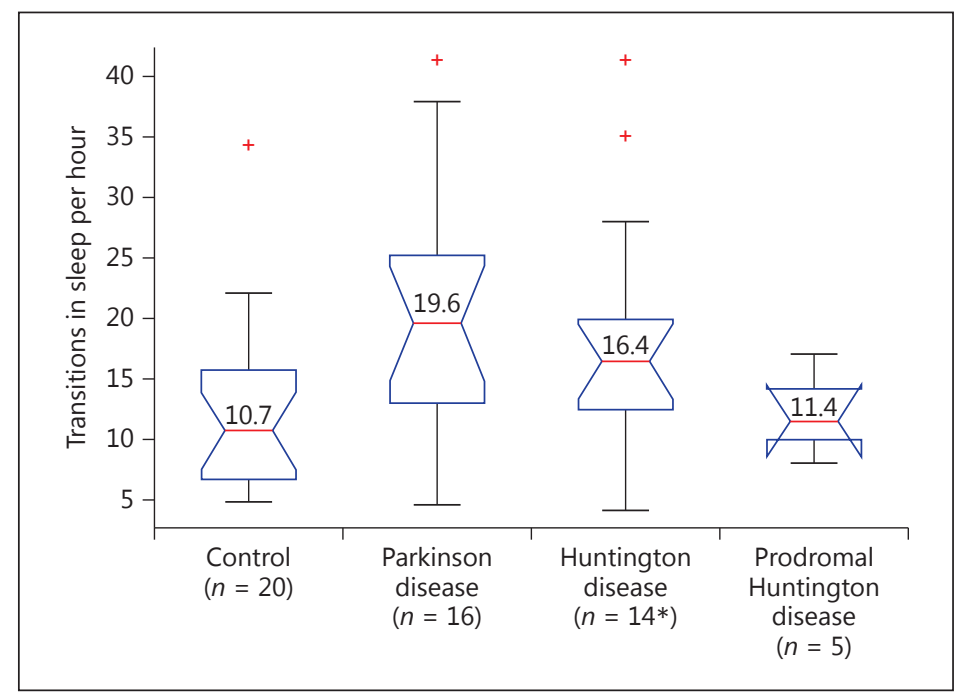

\section{Discussion}

In this small study, the use of self-adhesive wearable sensors among individuals with movement disorders was feasible, well-received, and identified new and important differences in activity levels among individuals with Huntington disease and Parkinson disease. Individuals with Huntington disease, all of whom were ambulatory, spent over half of the 48-hour observation period lying down, substantially more than any other group. This finding, which requires confirmation in future studies, is without precedent in the study of Huntington 
Adams et al.: Multiple Wearable Sensors in Parkinson and Huntington Disease Individuals

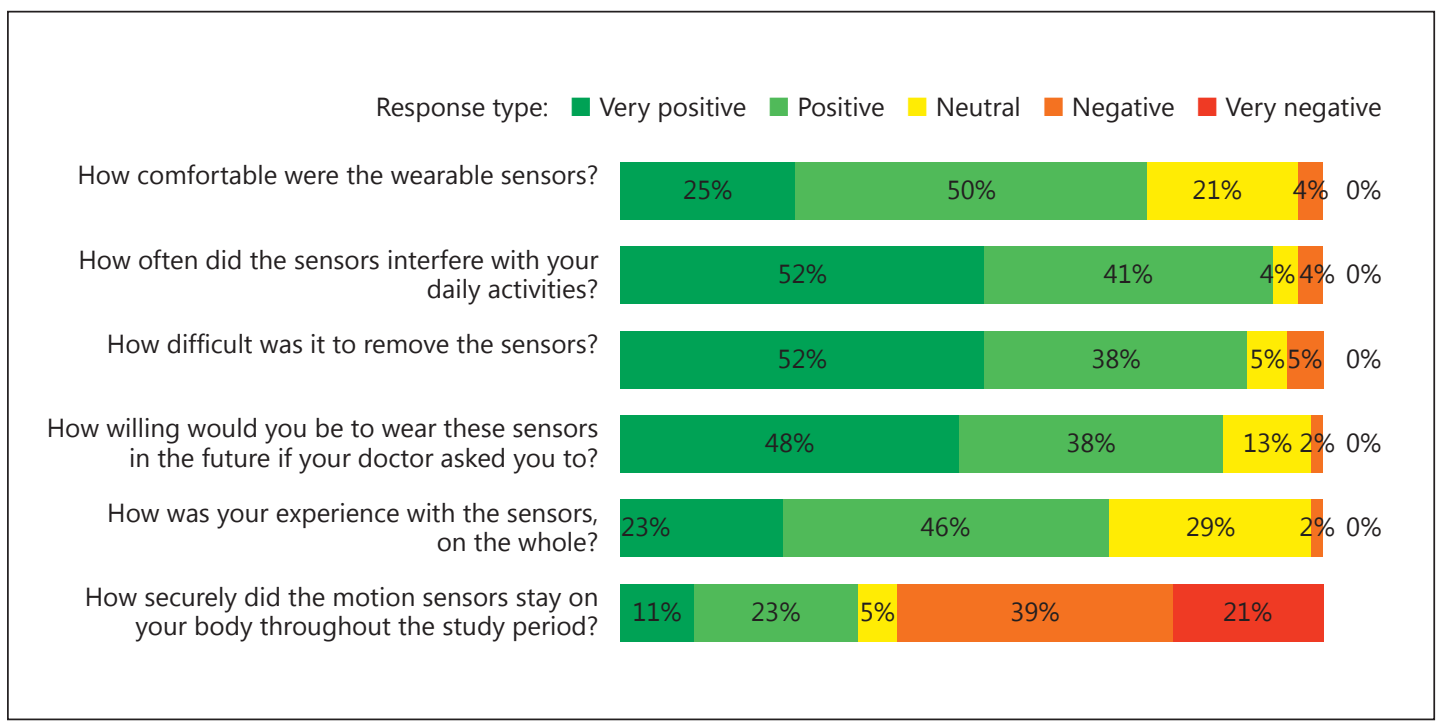

Fig. 5. Participant responses to end-of-study survey.

disease and leads to the hypothesis that the daily physical activity of those affected is markedly reduced.

One previous study evaluating energy expenditure in Huntington disease found that 24-hour sedentary energy expenditure and spontaneous physical activity were both increased among individuals with Huntington disease when compared to control individuals [18]. However, this spontaneous physical activity was highly correlated with severity of chorea, indicating increased energy expenditure due to involuntary movement rather than voluntary physsical activity. Previous studies have shown that in both prodromal and manifest Huntington disease populations, those with sleep disturbances have significantly worse neuropsychiatric outcomes compared to those without $[19,20]$. While it is known that disrupted sleep is an early symptom in individuals with prodromal Huntington disease, the underlying cause has not been identified and at-home monitoring has been limited [21]. Prior attempts to monitor sleep in the Huntington disease population using single and less sensitive activity monitors have shown poor agreement with EEG recordings and sleep diaries [22]. With our sensitive and comprehensive system of accelerometers, it may be possible to accurately monitor and characterize sleep disorders in the prodromal and manifest Huntington disease populations.

Individuals with Parkinson disease spent modestly more time lying down than individuals without a movement disorder. Previous studies have found that physical activity is reduced in individuals with mild to moderate Parkinson disease and that sedentary activity is increased in such individuals $[23,24]$. Another study using accelerometers in Parkinson disease found that sedentary behavior, defined as time spent lying or sitting, was similar among individuals with and without Parkinson disease, but that episodes of sedentary behavior were likely to be longer (and less frequent) among individuals with Parkinson disease [24]. Our findings support this premise as we observed that participants with Parkinson disease had fewer state transitions during the day than controls despite having similar activity levels. Although studies have shown that disease severity, gait difficulty, and disability in daily living contribute to inactivity in individuals with Parkinson disease, a part of the variance remains unexplained [25]. Wearable sensors can provide deeper insight into the extent and nature of changes in activity level due to disease progression and better characterize Parkinson-related sleep disorders. 
Adams et al.: Multiple Wearable Sensors in Parkinson and Huntington Disease Individuals

In this study, those with prodromal Huntington disease had activity profiles similar to controls. Larger sample sizes are required to confirm such findings, and other differences (e.g., in gait or involuntary movements) may still be present but were not part of this analysis. Identifying such differences could be helpful in finding outcome measures for assessing the efficacy of interventions aimed at individuals with prodromal Huntington (or Parkinson) disease.

This study is limited in its scale and scope. The number of study participants is modest, and the period of observation short (48 hours). Larger studies are required that include observation for perhaps briefer intervals but over longer time spans to determine how the measures in this study change over time. The scope of parameters assessed in this study was very narrow and focused on physical activity. Future analyses will investigate the presence of involuntary movements (e.g., tremor, chorea) and changes in gait among individuals in each of the groups. These analyses can also be used to gauge individual response to symptomatic therapy. Such objective analyses conducted in parallel with traditional assessments can provide an appraisal of the sensitivity of this new class of sensors in comparison to clinician-derived rating scales. This study also limited its assessment to data from accelerometers. These sensors and others include functions that can measure additional outcomes, including biometric data (e.g., heart rate, muscle activity), and thus provide additional insights. The analytical approach to assessing physical activity can be improved over time by utilizing video-recorded assessments in the home to give a more objective "ground truth" than diaries, and supply additional data to minimize the proportion of activities that are difficult to classify (e.g., sitting/standing).

Notwithstanding these limitations, this study uses multiple self-adhesive accelerometers to provide a new window to examining how movement disorders affect the activity of those with these conditions. This has the potential to expand our ability to assess patient symptoms beyond the traditional in-clinic assessments and lead to a deeper understanding of movement disorders and their impact on individuals. In addition, this emerging class of sensors provide new, relevant ways to measure disease course that can be used to assess existing and novel therapeutic interventions and to guide clinical care [26].

\section{Acknowledgements}

This study is supported in part by an unrestricted gift from MC10 Inc.

\section{Ethics Statement}

This study was reviewed and approved by the University of Rochester IRB.

\section{Conflict of Interest Statement}

Ray Dorsey is a consultant to MC10 Inc., a wearable device company, that provided the sensors used in this study. 
Adams et al.: Multiple Wearable Sensors in Parkinson and Huntington Disease Individuals

\section{Author Contributions}

J.L. Adams conducted study visits and contributed to writing the manuscript. K. Dinesh developed analysis methods, performed data analysis, and contributed to writing the manuscript. M. Xiong conducted study visits and contributed to writing/editing the manuscript. C.G. Tarolli conducted study visits and reviewed the manuscript. S. Sharma conducted study visits and reviewed the manuscript. N. Sheth contributed to the initial study design and reviewed the manuscript. A.J. Aranyosi contributed to data analysis and reviewed the manuscript. W. Zhu conducted study visits, contributed to initial study design, and reviewed the manuscript. S. Goldenthal conducted study visits and reviewed the manuscript. K.M. Biglan contributed to initial study design and implementation and reviewed the manuscript. E.R. Dorsey was the study's co-principal investigator and contributed to initial study design, implementation, and writing the manuscript. G. Sharma was the study's co-principal investigator and contributed to initial study design, implementation, and writing the manuscript.

\section{References}

1 Pentland A: "Sandy”. Social Physics: How Social Networks Can Make Us Smarter. Reissue. New York, Penguin Books, 2015.

2 Hird N, Ghosh S, Kitano H: Digital health revolution: perfect storm or perfect opportunity for pharmaceutical R\&D? Drug Discov Today 2016;21:900-911.

-3 Dorsey ER, Venuto C, Venkataraman V, Harris DA, Kieburtz K: Novel methods and technologies for 21st-century clinical trials. JAMA Neurol 2015;72:582.

4 Patel S, Lorincz K, Hughes R, et al: Analysis of feature space for monitoring persons with Parkinson's disease with application to a wireless wearable sensor system. 29th Annu Int Conf IEEE Eng Med Biol Soc, Lyon, 2007, pp 6290-6293.

5 Maetzler W, Domingos J, Srulijes K, Ferreira JJ, Bloem BR: Quantitative wearable sensors for objective assessment of Parkinson's disease. Mov Disord 2013;28:1628-1637.

-6 Ross CA, Tabrizi SJ: Huntington's disease: from molecular pathogenesis to clinical treatment. Lancet Neurol 2011;10:83-98.

7 Mannini A, Trojaniello D, Cereatti A, Sabatini A: A machine learning framework for gait classification using inertial sensors: application to elderly, post-stroke and Huntington's disease patients. Sensors 2016;16:134.

8 Andrzejewski KL, Dowling AV, Stamler D, et al: Wearable sensors in Huntington disease: a pilot study. J Huntingtons Dis 2016;5:199-206.

9 MC10. BiostampRC System. https://www.mc10inc.com/our-products/biostamprc (accessed February 3, 2017).

10 Nasreddine ZS, Phillips NA, Bedirian V, et al: The Montreal Cognitive Assessment, MoCA: a brief screening tool for mild cognitive impairment. J Am Geriatr Soc 2005;53:695-699.

$\checkmark 11$ Goetz CG, Tilley BC, Shaftman SR, et al: Movement Disorder Society-sponsored revision of the Unified Parkinson's Disease Rating Scale (MDS-UPDRS): scale presentation and clinimetric testing results. Mov Disord 2008; 23:2129-2170.

-12 Huntington Study Group: Unified Huntington's Disease Rating Scale: reliability and consistency. Mov Disord 1996;11:136-142.

13 Podsiadlo D, Richardson S: The timed “Up \& Go": a test of basic functional mobility for frail elderly persons. J Am Geriatr Soc 1991;39:142-148.

14 Duncan RP, Combs-Miller SA, McNeely ME, et al: Are the average gait speeds during the 10-meter and 6-minute walk tests redundant in Parkinson disease? Gait Posture 2017;52:178-182.

15 Zhan A, Little MA, Harris DA, et al: High frequency remote monitoring of Parkinson's disease via smartphone: platform overview and medication response detection. https://arxiv.org/pdf/1601.00960.pdf (accessed January 5, 2016).

16 Anton H, Busby RC: Contemporary Linear Algebra. New York, Wiley, 2003.

17 Dinesh K, Xiong M, Adams J, Dorsey R, Sharma G: Signal analysis for detecting motor symptoms in Parkinson's and Huntington's disease using multiple body affixed sensors: a pilot study. Proc IEEE West NY Image Signal Proc Work, Rochester, 2016.

18 Pratley RE, Salbe AD, Ravussin E, Caviness JN: Higher sedentary energy expenditure in patients with Huntington's disease. Ann Neurol 2000;47:64-70.

19 Baker CR, Domínguez D JF, Stout JC, et al: Subjective sleep problems in Huntington's disease: a pilot investigation of the relationship to brain structure, neurocognitive, and neuropsychiatric function. J Neurol Sci 2016; 364:148-153. 
Adams et al.: Multiple Wearable Sensors in Parkinson and Huntington Disease Individuals

20 van Wamelen DJ, Roos RA, Aziz NA: Therapeutic strategies for circadian rhythm and sleep disturbances in Huntington disease. Neurodegener Dis Manag 2015;5:549-559.

21 Lazar AS, Panin F, Goodman AOG, et al: Sleep deficits but no metabolic deficits in premanifest Huntington's disease. Ann Neurol 2015;78:630-648.

22 Townhill J, Hughes AC, Thomas B, etal: Using Actiwatch to monitor circadian rhythm disturbance in Huntington' disease: a cautionary note. J Neurosci Methods 2016;265:13-18.

23 Benka Wallen M, Franzen E, Nero H, Hagstromer M: Levels and patterns of physical activity and sedentary behavior in elderly people with mild to moderate Parkinson disease. Phys Ther 2015;95:1135-1141.

-24 Dontje ML, de Greef MHG, Speelman AD, et al: Quantifying daily physical activity and determinants in sedentary patients with Parkinson's disease. Parkinsonism Relat Disord 2013;19:878-882.

25 Nimwegen M, Speelman AD, Hofman-van Rossum EJM, et al: Physical inactivity in Parkinson's disease. J Neurol 2011;258:2214-2221.

26 Espay AJ, Bonato P, Nahab FB, et al: Technology in Parkinson's disease: challenges and opportunities. Mov Disord 2016;31:1272-1282. 